\title{
The needs of mothers to newborns hospitalised in intensive care units
}

\author{
Lucie Sikorova, Jana Kucova
}

\begin{abstract}
Objective. The aim of the survey was to identify the needs of mothers to infants hospitalized in intensive care units (ICUs) and second, to assess the level of parental support provided by the health personnel.

Methods. The sample consisted of 147 mothers to infants hospitalized in ICUs. The research was conducted over six months in ICUs for newborns at two hospitals in Ostrava. The study used two standardized questionnaires: The parental stressor questionnaire scale: Neonatal Intensive Care Unit which measures the degree of stress in parents of hospitalized infants and the questionnaire The Nurse Parent Support Tool which evaluates the level of parental support provided by nursing staff.

Results. The highest level of stress was identified in the parental role. Specifically, the inability to help the child remain separate from the mother, a feeling of helplessness and inability to protect the child from painful procedures and the inability to feed her baby. Mothers evaluated the support of nursing staff in most of these areas as high. Top were rated the ability of the caring staff to respond well to the questions of parents and the mother's willingness to engage in childcare.

Conclusion. Intensive care units for the newborn obviously need to be family-centered care and at the same time they must be aware of all the factors that can be sources of stress for the parents.Only in this way can stress be eliminated with positive impact on the relationship between mother and child.
\end{abstract}

Keywords: parental stressor scale, neonatal intensive care unit, the nurse parent support tool, stress

Received: May 11, 2011; Accepted: August 10, 2011; Available online: September 5, 2011 http://dx.doi.org/10.5507/bp.2011.046

Department of Nursing and Midwifery, Faculty of Medicine, University of Ostrava, Czech Republic Corresponding author: Lucie Sikorova, e-mail: lucie.sikorova@osu.cz

\section{INTRODUCTION}

Advances in medicine have led to increased levels of care for newborns. Modern technology and new knowledge in the field of neonatology have enabled babies on the edge of viability to be cared for and kept alive. However, this is a major emotional, psychological, social and financial burden on the family, especially parents. The medical personnel caring for such newborns too, are exposed to contact with parents and face different reactions from them which they have to manage effectively. Information on babies requiring special care, like this, is available in a large number of publications both nationally and internationally ${ }^{1}$. However, the needs of the parents of these neonates have not been published in the Czech literature. These parents must be understood as important team members in the contribution to the improved quality of life of their child in the long term.

\section{Intensive Care Units}

The environment of the intensive care unit for newborns (ICU) may appear stressful to the non-medical person. There is the movement of a large number of personnel, the continuous noise and characteristic smells and the instruments surrounding the child often make it impossible to be constantly with him/her. In the first week of the hospitalization of their child in the ICU, parents describe feelings of shock, apprehension and uncertainty about the health of their child. The ICU is for parents, a source of enormous tension. In mothers, this can reveal itself as depression and anxiety possibly due to ambiguity about their parental role, an uncertain prognosis and ineffective communication between parents and nursing personnel $^{2}$. Parents may be disappointed, they may have feelings of guilt, failure, despair, feelings of anger, helplessness and loss of self esteem. Hospitalization of the child may disrupt the relationship for a long time ${ }^{3}$. Sometimes it is necessary to deal with grief in parents after a failed pregnancy or unexpectedly difficult birth and disillusion ${ }^{4}$. Mothers may have a feeling of ambivalence, very often observed as mood swings between hope and hopelessness ${ }^{5}$. The task of neonatal and pediatric departments is to create appropriate conditions not only for children but also for their parents. Their participation in child care is indispensable. For parents, also desirable is support from the nursing staff. The expectations are emotional support, empowerment in decision making, a pleasant environment with a philosophy of support and the education of mothers through their participation in the care of the child ${ }^{6,7}$.

Based on the results of a number of research studies, important needs identified in parents are: security, care needs, communication,constant information, education, pain management, the need for proximity and support ${ }^{8}$. From analysis of research over the years 1998-2008, Cleveland ${ }^{9}$ identified 6 basic parental needs: a) accurate informatik, participation in child care and decision making, b) the care and protection of the child, c) contact with the child, d) positive receptive staff e) individual 
care, f), a calming and therapeutic relationship with nursing staff.

To identify the needs and assess the satisfaction of parents of hospitalized children a number of questionnaires and measurement tools such as the NIPS (NeonataI Index of Parent Satisfaction), PFQ (Parent FeedbackQuestionnaire), PSS: NICU (Parental stressor Scale: Neonatal Intensive Care Unit, NUPS (Neonatal Unit Parental Stress) and others have been developed internationally.

\section{MATERIALS AND METHODS}

The target group of the research were women whose children were hospitalized in intensive care units for newborns at the MNO or FNO in the first half of 2010 (MNO: $\mathrm{n}=61$ FNO: $\mathrm{n}=86$ ).

Selection criteria: Mothers hospitalized along with their child in the Intermediate Care Unit, at least one visit by the mother to the neonatal intensive care unit during hospitalization and mothers willing to participate in scientific research.

Exclusion criteria: Women who did not understand the Czech language.

\section{Aim}

The aim of this study was to determine the needs of mothers of newborns admitted to the neonatal intensive care units at the City Hospital in Ostrava (MNO) and the University Hospital of Ostrava (FNO). Specific objectives were to: identify stress factors in mothers of hospitalized newborns in four areas, namely visual stimuli and sounds, behavior apperance and medical treatment of the child, parental role and relationship with the child, the behavior of staff and communication and second, to assess the degree of support provided by nurses during the mothers' hospitalization of the newborn and determine the relationship between mothers'perception of stress apropos staff behavior and perceived support provided.

\section{Instruments}

Quantitative research using questionnaire surveys. Two standardized questionnaires were used: a questionnaire examining the levels of stress in parents of children admitted to neonatal intensive care units.

Parental stressor Scale: Neonatal Intensive Care Unit (the PSS:NICU) and a questionnaire exploring the support provided to the parents by the health Professional. The Nurse Parent Support Tool (theNPST). Both questionnaires were translated from the English original with double linguistic checking.

The questionaire PSS:NICU focuses on 4 areas: visual stimuli and sounds, behavior, appearance and medical treatment of the child, parental role and relationship with child and, the behavior of staff and communications.

The respondents answered a total of 37 items. This was a Likert response scale where 0 meant no experience with the described situation or phenomenon, 1 expressed no stress response and 5 , extreme stress in a given situation.

The questionnaire NPST. This questionnaire consists of 21 items. There was also a choice of answers on a Likert scale. Questions were aimed at the perceived support of the nursing staff. One meant a minimum support and 5 maximum support in different situations. The questionnaire was supplemented by respondents' demographic data and identification of selected infants.

\section{Data Processing}

Individual items of the questionnaires were first coded and processed using the statistical program Stata v. 10 .

Evaluation of stress levels corresponding to score points:

Low levels of stress from 1.10 to 2.59 points Likert scale. Median level of stress from 2.60 to 3.59 points Likert scale. High level of stress from 3.60 to 5.00 points Likert scale.

Rating of perceived support from healthcare professionals matching score points:

Low from 1.00 to 2.59 points Likert scale. Medium from 2.60 to 3.59 points Likert scale. High 3.60 to 5.00 points Likert scale.

Demographic data, perception of stress factors and levels of support provided to mothers were evaluated statistically using descriptive statistics. The authors of the questionnaire PSS: NICU describe more than one way of evaluating the data. One way is to evaluate the means and standard deviations of the questionnaire responses calculated from responses of those who had encountered the situations described. In this case, the responses of those who had no experience with that item, did not count. We chose this particular method of evaluation so that an answer 0 was not counted. With regard to data distribution and range of the Likert scale questionnaires, the results for the respondents on both medical instruments were tested using t-tests. To determine any dependence between the perception of support and perception of stress, the data from the NPST questionnaire were evaluated as an independent variable and data from the questionnaire PSS: NICU as a dependent variable. For determining any dependency between the two variables, we used the sum of the scores of individual items from both questionnaires. The relationship between the two areas was then assessed using a Spearman correlation coefficient. For multiple comparisons of data (level of maturity of neonate, stress levels in different areas) we used ANOVA and Bonferroni tests. All tests were evaluated for statistical significance at the level of $5 \%(0.05)$.

\section{RESULTS}

The majority of women were aged 21-30 years, $\mathrm{n}=73$ (49.7\%). Most were married $n=85(57.8 \%) .89$ (60.5\%) had secondary education. The pregnancy duration ranged from 23-42 weeks, on average 34 weeks. 21 women gave birth to twins. Newborns of the respondents had birth 
weights 480-5200g, mean birth weight was 2270 grams. The most common reason for hospitalization of infants was prematurity. Hospitalization of children in the ICU lastex from 1 to 120 days, on average 10 days. Mothers were hospitalized with their children at the time of completing the questionnaire an average of 5 days.

\section{Maternal stress factors}

The greatest levels of stress were found to be in the areas of parental role and relationship with the child. The average stress rating according to the results was 107 (72.8\%) of respondents with a high stress level. Another area which caused 61 (41.5\%) respondents high levels of stress was the behavior, appearance and medical treatment of the child (Table 1).

In the area of visual stimuli and sounds, most stressful identified by responders was the respiratory instruments around the child (medium stress level). Unexpected alarms of the monitor also caused medium levels of stress. Other stressful factors for this area (permanent noise of monitors and equipment, the presence of other patients, a large number of personnel, etc.) were assessed as low stress.

In the field-oriented behavior, the painful appearance of their child was assessed as highly stressful. Abnormal breathing, an unhappy expression, or fatigue and weakness also aroused a high degree of stress. Stress rated moderately were tremors and restlessness, cannulae and tubing around the child, inability of the baby to cry, its low weight, bruises or scars, the devices around the child, unusual color, infusions or feeding tubes.

In the area of parental role and relationship with the child several situations were identified that were a source of high stress levels in respondents: the impossibility of remaining away from the child, a feeling of helplessness and inability to protect the child from painful procedures and the inability to feed the baby. A medium level of stress was caused by inability to care for the child, to be alone with the baby and cuddle it.

In the area of personnel and communication behavior, mean levels of stress were found. Most respondents believed that the staff did not care for the presence of parents. The uncertainty about the staff informing them about any changes in the health status of the child, also caused medium stress. Another factor that contributed to a high stress level, was the concern that staff would understand the child's needs, provide various information, lack of communication from the staff or lack of information on the diagnosis and/or treatment. Informants met with difficulties in obtaining information or not understanding the information provided to them and how these experiences were a source of secondary stress. For the sake of clarity, individual stress factors were evaluated without distinction of areas in the questionnaires and ordered according to degree of stress from the most to the least stressful (Table 2).

\section{Evaluation of support provided to mothers by health personnel}

Mothers evaluated the support of nursing staff in most of these areas as high. Top was rated the good care of the baby, the ability of the child care staff to respond well to their questions, willingness to engage the mother in the childcare and early response to the needs of the child. The worst, rated as a medium level of support was the introduction of the staff to the parents, staff response to the needs of mothers, encouraging parents to ask questions, advising staff to comfort the baby, helping staff to talk about feelings, the inclusion of parents in discussion and appreciation of the mother's custody of the child.

\section{The relationship between the stress of communication and support staff}

The statistical evaluation confirmed an indirect linear relationship between variables. The relationship of stress in the communication field personnel and support staff was $r=-0.3926$, which means that positive behavior in the staff has the ability to eliminate the feelings feelings of stress in the mothers of hospitalized neonates.

\section{The relationship between stress in relation to parentel role and support staff}

No relationship between stress in relation to parental role and support from staff was found.The relationship was only $r=-0.1140$.

\section{Comparing the differences between FNO and MNO}

Comparing the differences in scores of individual items for respondents from the MNO and the FNO, greater stress was perceived by respondents from the

Table 1. Evaluation of stress levels.

\begin{tabular}{|c|c|c|c|c|c|c|}
\hline \multirow{2}{*}{ Maternal stress factors } & \multicolumn{2}{|c|}{ High level } & \multicolumn{2}{|c|}{ Median level } & \multicolumn{2}{|c|}{ Low level } \\
\hline & $\mathrm{n}$ & $\mathrm{n}(\%)$ & $\mathrm{n}$ & $\mathrm{n}(\%)$ & $\mathrm{n}$ & $\mathrm{n}(\%)$ \\
\hline Visual stimuli and sounds & 3 & 2.0 & 40 & 27.2 & 104 & 70.7 \\
\hline Infant behavior and appearance & 61 & 41.5 & 58 & 39.4 & 28 & 19.0 \\
\hline Parental role & 107 & 72.8 & 29 & 19.7 & 11 & 7.5 \\
\hline Personnel and communication behavior & 31 & 21.1 & 30 & 20.4 & 53 & 36.0 \\
\hline
\end{tabular}


Table 2. Individual stress factors (The questionaire PSS:NICU).

\begin{tabular}{lc}
\hline \multicolumn{1}{c}{ High level } & Median \\
\hline Feeling helpless about how to helpmy baby during this time & 4.4 \\
Being separated from my baby & 4.2 \\
Feeling helpless and unable to protect my baby from pain and painful procedures & 4.2 \\
When my baby seemed to be in pain & 4.1 \\
My baby's unusual or abnormal breathing patterns & 4.0 \\
When my baby looked sad & 4.0 \\
Not feeding my baby myself & 3.6 \\
The limp and weak appearance of my baby & 3.6 \\
\hline
\end{tabular}

Median level

\begin{tabular}{lr}
\hline Jerky or rest less movements of my baby & 3.5 \\
Seeing needles and tubes put in my baby & 3.5 \\
Having a machine (respirator) breathe for my baby & 3.5 \\
My baby not beingable to crylike other babies & 3.5 \\
Staff acting as if they didn't want parents around & 3.5 \\
The suddennoisesof monitor alarms & 3.5 \\
Not feeling sure that I will becalled about changes in my baby's condition & 3.5 \\
Not beingable to care for my baby myself (for example, diapering,bathing) & 3.5 \\
Not beingable to bealone with my baby & 3.4 \\
Staff acting as if they did not understand my baby's behaviour or special needs & 3.4 \\
Telling me different things about & 3.3 \\
Not talking to meenough & 3.3 \\
Not beingable to hold my baby when I want & 3.3 \\
Bruises, cutsorincisions on my baby & 3.3 \\
Tubes and equipment on or near my baby & 3.1 \\
Not telling me enough about tests and treatments being done to my baby & 3.1 \\
Staff using words I don't understand & 2.8 \\
My baby beingfed by an intravenousline or tube & 2.8 \\
Difficulty in getting information or help when I visit or telephone & 2.6
\end{tabular}

Low level

\begin{tabular}{lr}
\hline Staff explaining things too fast & 2.5 \\
The constant noises of monitors and equipment & 2.3 \\
The presence ofmonitors and equipment & 2.2 \\
The wrinkled appearance of my baby & 2.2 \\
Too many different people talking to me & 2.0 \\
The others ick babies in the room & 1.8 \\
The large number of people working in the unit & 1.3
\end{tabular}

University Hospital. The exceptions were only three items in the questionnaire PSS: NICU,which were evaluated at a higher average level of stress for respondents from MNO. The difference between their evaluation and that of the respondents from the University Hospital was negligible for two items, 0.1 points. The only item with a larger difference in rating, 0.8 points, was due to difficulty in obtaining information from respondents at the MNO. For respondents from the University Hospital a greater source of stress, compared to respondents from MNO, was in the area of the information. A significant difference in rating was found to be misunderstanding information, different information provided by different staff members and lack of information. The the evaluation using a $\mathrm{t}$ - test confirmed a statistically significant difference between the level of support provided to mothers by nursing staff in the two hospitals. Respondents assessed the support at the MNO better than respondents in the University Hospital $(\mathrm{p}=0.0144)$.

\section{DISCUSSION}

Based on the data analysis, it was revealed that for mothers of newborns admitted to neonatal iintensive care units, the most stressful areas are the parental role and 
relationship with the child. Here mothers showed high levels of stress. For behavior, appearance, and treatment of the child and the behavior of staff and communication there was a moderate degree of maternal stress. For visual stimuli and sound, this was assessed as causing low levels of maternal stress.

These findings partly correlate with the results of Reid and Bramwell ${ }^{10}$ who studied a sample of British mothers and found the same results for behavior, appearance and medical treatment of the child but a slightly higher average stress values in the evaluation of the areas of visual stimuli and sounds and parental roles. Authors of research done in New Zealand, also came to similar conclusions ${ }^{11}$. Also, they identified the most stressful area as parental role and the least stressful area as staff behavior and communication. The area of parental role, other authors as well have identified as a major source of stress in mothers of preterm neonates ${ }^{12,13}$.

Similar conclusions were drawn by authors of research conducted in the U.S. and Great Britain on a sample of 257 parents. Their results showed a moderate degree of stress in their parental role and relationship with the child (mean 3.4) and in behavior, appearance, and the child's medical procedures (average 3.2). Low levels of stress were assessed in the area of staff behavior and communication (average 2.6 in the U.S., and 2.2 in Great Britain) and the visual stimuli and sounds (in the U.S. the average was 2.3 and 2.3 in Great Britain) ( $\operatorname{ref}^{14}$ ). Comparing the sum of points in the various fields of the research questionnaires PSS: NICU with with the results of longitudinal studies in Australia investigating stress in parents of preterminfants, we found similar results in three areas of the questionnaire PSS: NICU. Only in the area of the behavior of staff and communication was there a distinct difference. The authors describe a comparison study in a group of mothers at a hospital in Melbourne, Australia and they found a significantly lower level of stress in this area (the average 8.6) $\left(\right.$ ref $\left.^{15}\right)$.

As not all of our survey respondents had experience with some of the situations in different areas, it follows, inter alia, that in those areas, the mothers were satisfied as they did not meet those factors at all.This is good especially in the area of parental role and relationship with the child in areas related to behavior and communication with personnel. If parents in these areas had no experience, then this could only mean that the staff considered the parents as parents, able to participate in the care of the child and had no problems with communication.

Another aim of our investigation was to evaluate the level of support provided to mothers during hospitalization by nurses. Although respondents were largely positive, in some areas we found significant differences between respondents' assessment and the assessment in the research carried out in the Children's Hospital at Westmead, Australia. Comparing the average rating for each item on the questionnaire NPST for both research surveys, the most pronounced difference was found in the area of introductions to staff, staff response to the needs of parents and encouraging questions from the parents by the nursing staff ${ }^{16}$. In foreign countries, parental support in these areas is more commonplace. This finding is almost identical to the results of studies in the general hospital of Hong Kong, where three areas were rated very similarly. Comparing the overall average results of the evaluation questionnaire NPST for the hospitals in Ostrava (average 3.8) with the average from the research in Hong Kong (mean 3.8) there is agreement ${ }^{17}$. In contrast, research at the Iranian Children's Hospital suggests stronger support of nursing personnel expressed by respondents in Iran. Here, the average score was 4.1 points $^{18}$.

Analysis of the type and degree of relationship between the two variables monitored (the relationship between mothers'perceptionof stress and staff behavior and perceptions of support provided to mothers by nurses), showed an an indirect linear relationship. This suggests that the perceived stress in the area of in staff behavior and communication can be influenced by the support provided by nursing staff. Such a finding correlates with the results of research at the Children's Hospital at Westmead ${ }^{19}$.The difference in the value of the correlation coefficient was probably due to lower levels of stress perceived by mothers in the Ostrava region in both hospitals. The support of the nursing staff was evaluated by both survey respondents alike.

Analysis of the relationship between mothers' perception of stress in the area of the behaviour of staff and perception of support provided to mothers by the nurses revealed no dependency. It seems that the respondents' experienced stress in the area of parental role and relationship with the child was relatively high, despite the positive assessment of the nursing team. We believe that even though mothers of hospitalized neonates cannot participate in the care of the child in the normal way, they can establish other ways of contact with the child. For example, regular infusion of incubator baby milk, baby massage or kangaroo mother care. These forms of assistance as alternative ways to establish a positive relationship between mother and child have been shown by other authors ${ }^{20}$. Mothers can get a sense that they are partners in caring for the child.

Differences in perception of stress between FNO a MNO in the area of behavior, appearance and medical treatment of the child may be explained by the differences in patients, larger equipment and instrumentation providing highly specialized care for newborns at the University Hospital. Parents can see their children far more often than parents of infants hospitalized in the MNO, the child is connected to different devices, with cannulae and the child's condition is often critical. This was clear from the number of respondents at the University Hospital who had met with or experienced the situation described by the questionnaire.Their scores were on almost all items higher than the scores of respondents from the MNO.

In the area of staff behaviors and communication we cannot ignore the difference in evaluation. More stress was experienced by respondents from FNO especially in areas related to communication. These research results 
are confirmed by Italian authors who assessed the degree of parental stress in relation to the various options for communication with parents, staff, or ICU time of visit ${ }^{21}$. Since both neonatal ICUs, where the research took place have almost unlimited visits and organization of information, both personal and telephonic, were very similar, we assumed conformity in assessment of stress levels in staff behavior and communication at both hospitals. It is understandable that parents when they first visit the baby in the ICU experience feelings of shock, disappointment and fear. For this reason, they often do not remember information. Therefore, this should be done again on subsequent visits. Nursing staff who manage the technology education should be able to identify areas in which parents need more information. Feedback is then necessary that makes it possible to check their understanding of the information provided. It is therefore very important to encourage parents to ask questions. From the research it is evident that this form of support was rated as one of the worst. However, there should be consistency in the information provided by the nursing team. It is very important for effective mutual communication. These simple interventions would reduce the future occurrence of stress factors in parents of hospitalized newborns in the field of communication. It must be emphasized however that not all mothers had experience of stress in this area.

After analyzing the data, it can be stated that while the nursing staff in MNO usually took parents for granted, at the University Hospital in this area there is still some reserve. The anonymity of the nursing staff is now no longer taboo. Even though the names of the nursing staff can be read on their identity cards, each team member should introduce herself/himself personally to the parents of the newborn. And despite the fact that parents in the first days of hospitalization, were provided with alot of information, they should be shown to be a permanent part of the organizational culture in both hospitals.

For the same reasons, the evaluation of the respondents also indicate that the MNO staff involve parents in child-care more often than FNO. These differences can be explained by the different care provided by the ICUs. While the neonatal intensive care unit for newborns in the MNO provides for intermediate care, the intensive care unit for newborns at the University Hospital provides for intensive care and resuscitation. For this reason, the lower the type of work and more opportunities to involve mothers in caring for their child and allowing them to decide how to care for their baby are more available in the MNO.

For the same reason, we more often found respondents from the MNO able to decide whether a child would have or not have a medical procedure.The FNO babies are often hospitalized with more serious diagnoses, and must observe strict asepsis during demanding procedures. The Newborn Intensive Care Unit in the MNO has a variety of hospitalized infants in a less dire condition. The babies are not extremely endangered, and the mothers can stay with the baby for most procedures. Also, the size of the ICU room allows the presence of parents in the work of the nurses. Access to staff at both hospitals in this area should remain individual.

\section{CONCLUSIONS}

The significance of this investigation was identification of specific factors contributing to stress in parents/ mothers of infants admitted to ICUs and in identifying areas of insufficient support provided by the nursing team. Knowledge of these factors can facilitate the development of intervention strategies primarily in relation to the parents. Staff should select a single plan. This should include support to parents by the ICU staff in all areas described. At the same time there is a need to adapt to the individuality of each family. Application of knowledge to practice can influence the attitude of parents and parent satisfaction with the care provided by nursing staff. Support should reduce stressful influences. If these needs are met, we can expect improved communication between mother and child and between mother and health care professionals.

\section{ABBREVIATIONS}

ICU, Intensive Care Unit; NIPS, NeonataI Index of Parent Satisfaction; PFQ, Parent FeedbackQuestionnaire; PSS: NICU, Parental Stressor Scale: Neonatal Intensive Care Unit; MNO, City Hospital in Ostrava; FNO, University Hospital of Ostrava; NUPS, Neonatal Unit Parental Stress

\section{REFERENCES}

1. Tomankova L. Péče o novorozence na vlnách času aneb pohled do historie neonatologie. Cesk Slov Pediat 2006;6:76-83.

2. Hayes JS, Ward K. Perceived Needs of Parents of Critically III Infants in a Neonatal Intensive Care Unit. Paediatr Nurs 2001;27:281-6.

3. Franck LS, Cox S, Allen A, Winter I. Measuring neonatal intensive care unit-related parental stress. J Adv Nurs 2005;49:608-15.

4. Franklin C. The Neonatal Nurse's Role in Parental Attachment in the NICU. Crit Care Nurs 2006;29:81-5.

5. Obeidat HM, Bond EA, Callister LC. The Parental Experience of Having an Infant in the Newborn Intensive Care Unit. J Perinat Educ 2009;18:23-9.

6. Cleveland LM. Parenting in the Neonatal Intensive Care Unit. J Obstet Gynecol Neonatal Nurs 2008;37:666-91.

7. Franck LS, Cox S, Allen A, Winter I. Measuring neonatal intensive care unit-related parental stress. J Adv Nurs 2005;49:608-15.

8. Conner JM, Nelson EC. Neonatal Intensive Care: Satisfaction Measured From a Parent's Perspective. Pediatrics 1999;103(1 Suppl E):336-49.

9. Cleveland LM. Parenting in the Neonatal Intensive Care Unit. Obstet Gynecol Neonatal Nurs 2008;37:666-91.

10. Reid T, Bramwell R, Booth N, Weindling AM. A new stressor scale for parents experiencing neonatal intensiv care: the NUPS (Neonatal Unit Parental Stress) scale. J Reprod Infant Psychol 2007;25:66-82.

11. Carter JD, Mulder RT, Darlow BA. Parental stress in the NICU: The influence of personality, psychological, pregnancy and family factors. Personal Ment Health [serial on the internet]. 2007 Jan [cited 2010 Oct 17]. Available from: http://www.onlinelibrary.wiley.com/ doi/10.1002/pmh.4/pdf.

12. Trombini E, Surcinelli P, Piccioni A, Alessandroni R, Faldella G. Environmental factors associated with stress in mothers of preterm newborns. Acta Paediatr 2008;97:894-7.

13. Akbarbegloo M, Valizadeh L. Comparison of Mothers and Nurses Viewpoint about Stressors for Parents with Premature Newborn in NICU. Research J Biol Sci 2009;4:796-9. 
14. Franck LS, Cox S, Allen A, Winter I. Measuring neonatal intensive care unit-related parental stress. J Adv Nurs 2005;49:608-15.

15. Lau RGL. Stress Experiences of Parents with Premature Infants in a Special Care Nursery. Victoria University, Faculty of Human Development. 2001 [cited 2011 Jan 13]. Available from: http://eprints. vu.edu.au/228/.

16. Lam J, Spence K, Halliday R. Parents' perception of nursing support in the neonatal intensit care unit (NICU). Neonatal, paediatric and child health nursing 2007;10:19-25.

17. Mok E, Leung SF. Nurses as providers of support for mothers of premature infants. J Clin Nurs 2006;15:726-34.
18. Sanjari M, Shirazi F, Heidari S, Salemi S, Rahmani M, Shoghi M. Nursing Support for Parents of Hospitalized Children. Issues Compr Pediatr Nurs 2009;32:120-30.

19. Lam J, Spence K, Halliday R. Parents' perception of nursing support in the neonatal intensit care unit (NICU). Neonatal, pediatric and child health nursing 2007;10:19-25.

20. Cleveland LM. Parenting in the Neonatal Intensive Care Unit. Obstet Gynecol Neonatal Nurs 2008;37:666-91.

21. Trombini E, Surcinelli P, Piccioni A, Alessandroni R, Faldella G. Environmental factors associated with stress in mothers of preterm newborns. Acta Paediatr 2008;97:894-7. 